\title{
Thermoelastic investigation of residual stress: plastic deformation and the change in thermoelastic constant
}

\author{
A. F. Robinson ${ }^{1}$, J. M. Dulieu-Barton ${ }^{1}$, S. Quinn ${ }^{1}$, R. L. Burguete ${ }^{2}$ \\ ${ }^{1}$ University of Southampton, School of Engineering Sciences, Highfield, Southampton, SO17 1BJ, UK \\ ${ }^{2}$ Airbus Operations Ltd., New Filton House, Filton, Bristol, BS99 7AR, UK
}

\begin{abstract}
Plastic deformation causes very small changes in the thermoelastic response of metallic materials; this variation of the thermoelastic constant has the potential to form the basis of a new non-destructive, non-contact, full-field technique for residual stress assessment that is quicker and cheaper than existing methods. The effect of plastic strain on the thermoelastic constant is presented as a potential basis for a calibration methodology that reveals areas of a component that have experienced plastic strain. Establishing this basis provides the initial step in identifying a new approach to residual stress analysis using the thermoelastic response. An evaluation of initial calibration results is presented and the feasibility of applying the methodology to actual components is assessed. As the response to plastic strain is likely to be small it is necessary to identify the effects of the paint coating; experimental work is presented that highlights the importance of repeatable coating approaches.
\end{abstract}

\section{Introduction}

Residual stresses may be introduced into a component throughout its entire manufacturing process; it is highly unlikely that an in-service component is free of residual stresses. Since residual stresses are an almost unavoidable bi-product of manufacture, it is important to understand how residual stresses are distributed in a component to define its performance characteristics. At present, there are several techniques available for measuring residual stresses. However, destructive methods are not always practical for an in-service industrial environment, while the non-destructive methods are typically expensive and time consuming. Therefore, demand for a cheaper and quicker non-destructive, noncontact, full-field residual stress evaluation technique is increasing.

Thermoelastic stress analysis (TSA) [1] has been identified as a possible solution for a robust and portable means of non-destructive residual stress evaluation. TSA is a well established noncontacting analysis technique that provides full-field stress data over the surface of a cyclically loaded component. It is based on the small temperature changes that occur when a material is subject to a change in elastic strain, generally referred to as the 'thermoelastic effect'. When a material is subjected to a cyclic load, the induced strain produces a cyclic variation in temperature. The temperature change $(\Delta T)$ can be related to the change in the 'first stress invariant', $\Delta\left(\sigma_{1}+\sigma_{2}+\sigma_{3}\right)$,

\footnotetext{
${ }^{1}$ e-mail : a.robinson@soton.ac.uk
} 
or the sum of the principal stresses [1]. An infra-red detector is used to measure the small temperature change, which can then be related to the stress using the following equation:

$$
\Delta T=-K T_{0} \Delta\left(\sigma_{1}+\sigma_{2}+\sigma_{3}\right)
$$

where $T_{0}$ is the absolute temperature and $K$ is the thermoelastic constant, $K=\alpha /\left(\rho C_{p}\right)$, where $\alpha, \rho$, $C_{p}$ are the material constants of the coefficient of thermal expansion, mass density and the specific heat at constant pressure, of the material respectively.

As residual stress is essentially a mean stress, it is accepted that the linear form of the TSA relationship given in equation (1) does not allow its evaluation. However, there are situations where this linear relationship is not valid and these have enabled evaluations of residual stresses. Small variations in the thermoelastic response, resulting from the temperature dependence of the elastic properties, permitted the measurements. However, these changes in the thermoelastic response result in measured temperature change differences of a few $\mathrm{mK}$, which are significantly less than those expected to be resolved in standard TSA. It was shown [2] that a revised form of the thermoelastic equation relating the rate of temperature change and the rate of change in stress can be written as:

$$
\dot{T}=\frac{T_{0}}{\rho C_{p}}\left[-\left(\alpha+\left(\frac{v}{E^{2}} \frac{\partial E}{\partial T}-\frac{1}{E} \frac{\partial \boldsymbol{v}}{\partial T}\right) \sigma_{k k}\right) \dot{\sigma}_{k k}+\left(\frac{(1+v)}{E^{2}} \frac{\partial E}{\partial T}-\frac{1}{E} \frac{\partial \boldsymbol{v}}{\partial T}\right) \sigma_{i j} \dot{\sigma}_{i j}\right]
$$

where $\dot{T}$ is the rate of change of temperature, $E$ is Young's modulus of the material, $\boldsymbol{v}$ is Poisson's ratio and $\sigma_{i j}$ is the stress tensor. Dotted terms in Equation (2) represent a rate of change. Essentially $\sigma_{k k}$ is the mean stress, and $\dot{\sigma}_{k k}$ is the rate of change of the first stress invariant with respect to time.

Equation (2) differs from the Equation (1) in that the elastic constants are not assumed to be temperature independent. It can be seen in Equation (2) that the temperature response is actually dependent on the stress rate, as well as the stress state, i.e. the mean stress.

At present, three approaches have been investigated as potential candidates for residual stress measurement using the thermoelastic response [3]. Two are based on the mean stress effect and the revised higher order theory of Equation (2). One utilises the thermoelastic response at the second harmonic of the loading frequency [4], and the other directly relates the change in the thermoelastic response to the principal stresses [5]. The major limitation of these two approaches is that they are not suitable for steel components since the temperature dependence of the elastic properties of steel are negligible at room temperature [3]. The third approach [6] is based on Equation (1) and the change in the thermoelastic constant, $K$, resulting from plastic deformation during manufacture or assembly. In the third approach the main disadvantage is that plastic deformation must have taken place, but it has the advantage that it may be valid for a larger range of materials, not just those with temperature dependent elastic properties. A significant disadvantage common to all three approaches is that any change in the thermoelastic response resulting from either $\sigma_{m}$ or from the modification of $K$ will be small. In actual components the changes in the response are around the noise floor of the detectors. Success in detecting these changes has been achieved by applying very large residual stress or plastic strain, or by using materials that are very sensitive to the mean stress effect. Recently the sensitivity of infra-red detectors has improved to the extent where it may be possible to accurately measure changes representative of those in actual components, hence leading to a renewed interest using TSA for residual stress analysis. Since the variations in thermoelastic response are significantly smaller than the changes that are resolved during standard TSA, it is important to minimise sources of signal attenuation. As metallic specimens require a high emissivity 
coating to be applied to the surface which is known to cause attenuation [7] in the response, the effect of the coating must be considered in parallel to evaluations of the significance of changes in the response due to $\sigma_{m}$ or from the modification to $K$.

In the present paper the focus is on the third approach, described in [6]. Changes in the thermoelastic constant, $K$, and the thermoelastic constant for different levels of plastic strain, $K_{P}$, for stainless steel $316 \mathrm{~L}$ are identified. This is an important initial step for determining a calibration methodology for different levels of plastic strain. Essentially a plastic strain is applied to test specimens by loading into the plastic region and then unloading, with the purpose of defining $K_{P}$ for a range of plastic strain by conducting TSA on the specimens. Finally, the feasibility of applying the methodology to actual components with realistic levels of plastic strain is assessed. Here a different material is used so that it can be established if the effect is strong enough in materials other than steel, and to establish the effect of paint coating on a material with a different base emissivity. Therefore aluminium plates with cold expanded holes representative of those used in the aircraft industry were examined. Cold expanded holes have a relatively well defined residual stress distribution, thus, the potential of the approach can be considered in this exercise.

\section{Methodology for establishing the effect of plastic deformation on the thermoelastic constant}

It has been shown [6] that the introduction of plastic deformation modifies the thermoelastic constant in some metals due a change of the material properties contained in $K$. It has been suggested that this change in thermoelastic constant can be used to estimate the level of plastic strain that a component has been subjected to. Finally, since plastic strain can be related to residual stress, there is an opportunity to derive a procedure for the assessment of residual stress using TSA, utilising the effect of plastic deformation on the thermoelastic constant. Rosenholtz et al [8], and Rosenfield et al [9] have both demonstrated that in steel and aluminium, an application of plastic strain will cause a change in the material property, $\alpha$, the coefficient of linear thermal expansion. Rosenfield et al [9] also noted that this change in $\alpha$ increases significantly when subjected to compressive strains, and less with tensile plastic straining.

The experimental approach adopted in this work is to obtain the thermoelastic constant using TSA and compare it to a known value either calculated from material properties, or from a reference or calibration specimen. To indicate if plastic deformation causes a change in thermoelastic constant, a specimen can be loaded in uniaxial tension. The equation for the 'revised' thermoelastic constant for a specimen loaded in uniaxial tension is given by:

$$
K^{\prime}=\frac{1}{\rho C_{p}}\left[\alpha-\left(\frac{1}{E^{2}} \frac{d E}{d T}\right) \sigma_{m}\right]
$$

where $\sigma_{m}$ is the mean applied stress and $K^{\prime}$ is a revised thermoelastic constant.

Equation (3) enables $K^{\prime}$ and the effect of $d E / d T$ to be defined using material properties. It can be seen that the $d E / d T$ term must be considerable in magnitude if the mean stress is to have any significant influence on the thermoelastic constant. The mean stress effect in steel is very small, and it is has been demonstrated recently to be negligible in stainless steel [10]. This is because $d E / d T$ is very small, and thus even if there is a significant mean stress there will be a negligible effect on the thermoelastic response. 
In the experiments used in the present work, tensile strip specimens loaded in uniaxial tension are used for the following three reasons:

(i) If this type of specimen is loaded beyond the material's yield point and then unloaded, it will result in a residual strain; however, there will be no residual stress as the stress can be fully relaxed by the elastic unloading. Without the contribution of a residual stress that would result in an increase in $\sigma_{m}$ when loaded, any change in the thermoelastic response would be due to a change in one of the material properties, $\alpha, \rho$ or $C_{p}$.

(ii) In a dynamically loaded tensile specimen, non-adiabatic conditions cannot occur because there is no stress gradient [11], and therefore no heat transfer within the specimen. The only heat transfer is caused by the coating.

(iii) The stress in a specimen loaded uniaxially can be calculated in a straightforward manner. If $T$ and $\Delta T$ can be measured, and given that the loading conditions are defined, an experimental $K$ can be directly calculated using Equation (1).

A Cedip Silver $480 \mathrm{M}$ infra-red detector system was used to obtain $T$ and $\Delta T$, thereby enabling a direct calculation of the thermoelastic constant to be performed since the applied stress is known. This calculated value of the thermoelastic constant can then be compared to a value obtained analytically from material properties using Equation (3). This approach is taken to validate the thermoelastic data, and to enable a comparison of the thermoelastic constant for different levels of plastic strain, paint thickness, loading frequency and mean stress. Material properties used for the analytical calculation of the thermoelastic constant for stainless steel 316L and 7085-T7651 are shown in Table 1 [12]. It is clear that $d E / d T$ is finite for the stainless steel and removing the possibility of any residual stress changing the equivalent mean stress by using specimens loaded in uniaxial tension is appropriate. It is clear that for the 7085-T765 aluminium alloy plates with cold expanded holes, there is a possibility of the mean stress having an effect.

Table 1: Material properties of aluminium and stainless steel used for calculating the thermoelastic constant, $K$

\begin{tabular}{|l|c|c|}
\hline Material Property & AL7085 & 316L \\
\hline Coefficient of thermal expansion, $\alpha, \times 10^{-6}{\mathrm{~m} \cdot \mathrm{m}^{-1} \mathrm{~K}^{-1}}^{-3}$ & 23.6 & 16.2 \\
\hline Density, $\rho, \mathrm{kg} \mathrm{m}^{-3}$ & 2770 & 8000 \\
\hline Elastic modulus, $E$, GPa & 71 & 193 \\
\hline Specific heat, $C_{p}, \mathrm{~J} \mathrm{~kg}^{-1} \mathrm{~K}^{-1}$ & 875 & 500 \\
\hline Temperature dependence of elastic modulus, $d E / d T, \mathrm{MPa} \mathrm{K}{ }^{-1}$ & $-36[14]$ & $-19[10]$ \\
\hline Thermoelastic constant, $K, \times 10^{-12} \mathrm{~Pa}^{-1}$ & 9.73 & 4.05 \\
\hline
\end{tabular}

Adopting the approach described above is supported by the findings of Quinn et al [6], where a similar procedure was used on mild steel specimens that had experienced different levels of plastic strain; one specimen was left unstrained, while three specimens were statically strained to give maximum tensile strains of $5 \%, 6 \%$ and $8 \%$, and then unloaded. The change in $K$ was small, but it was seen to increase linearly with the level of plastic strain experienced. The effect was shown to be repeatable. Furthermore, if this change in thermoelastic constant can be accurately measured and compared to an unstrained calibration specimen, the plastic strain, and potentially the residual stress experienced by the component could be derived. In later work it was concluded that the change in thermoelastic constant was dependent on the material dislocation that occurs during strain hardening [13], and that the change in $K$ for a material that does not strain harden would be significantly less than for a material that does. 


\section{The effect of plastic deformation on the thermoelastic constant for stainless steel $316 \mathrm{~L}$}

Tensile specimens were manufactured using a wire erosion technique to ensure that residual stresses were not introduced during manufacture procedure. Each specimen had a nominal crosssectional area of $2 \mathrm{~mm} \times 18 \mathrm{~mm}$ and was coated with two passes of RS matt black paint [15], corresponding to a paint thickness of approximately $20 \mu \mathrm{m}$. An Instron 5500 electro-mechanical test machine was used to impart $2 \%$ and $4 \%$ plastic strain into two of the specimens; a third specimen was left with $0 \%$ plastic strain. The plastic strain levels were achieved in three steps: (i) $0.5 \mathrm{~mm} / \mathrm{min}$ extension until yield, (ii) $0.5 \mathrm{~mm} / \mathrm{min}$ until an additional $2 \%$ (or $4 \%$ ) strain, (iii) $-0.5 \mathrm{~mm} / \mathrm{min}$ until initial load. An extensometer with a $25 \mathrm{~mm}$ gauge length was used to measure and control the strain during the test. Stainless steel 316L was chosen for its reported large strain hardening ability; subsequent tensile tests have revealed that it strain hardens by approximately $50 \%$.

In the thermoelastic work, an Instron 8800 servo-hydraulic test machine was used to cyclically load the specimens at a load amplitude of $\pm 2 \mathrm{kN}( \pm 57.5 \mathrm{MPa})$ about a mean load of $3 \mathrm{kN}(86 \mathrm{MPa})$; thermoelastic data was recorded using the Cedip system at loading frequencies of 5 to $15 \mathrm{~Hz}$ in increments of $2.5 \mathrm{~Hz}$ to assess the effect of paint thickness on results.

A normalised thermoelastic constant, $K_{p} / K$, is defined where $K$ is the thermoelastic constant from the specimen without plastic strain. In Figure $1 \mathrm{a}, K_{p} / K$ is plotted for different loading frequencies for 316L stainless steel. The $K_{p} / K$ is shown to increase with increasing plastic strain; the largest increase at $2 \%$ strain is similar to the smallest increase at $4 \%$ strain. The same specimen was repainted with three passes of RS matt black (corresponding to approximately $30 \mu \mathrm{m}$ ) and the results shown in Figure $1 \mathrm{~b}$; in some cases, a decrease in normalised thermoelastic constant is seen between $2 \%$ and $4 \%$ plastic strain, however, there remains an increase from the $0 \%$ reference value. It is important to minimize any signal attenuation as much as possible. The disparities due to cyclic loading frequency highlight the difficulties posed by the paint coating.

In previous work [15] it was shown that as paint thickness and loading frequency increase, there is a decrease in the thermoelastic signal due to the coating effects of thermal lag and thermal dragdown. As seen in Equations (1) and (2), the loading frequency should have no effect on the thermoelastic response; Figure 1c shows the change in thermoelastic response due to loading frequency and the reduction in the thermoelastic constant is a result of a lower $\Delta T$ value measured at the surface of the specimen. This attenuation is caused entirely by the paint coating. Figure 1d further highlights the effect of paint coating thickness and loading frequency in a study focused on mild steel (AISI 1016) specimens. The expected thermoelastic constant for AISI 1016 is $3.04 \times 10^{-12}$ $\mathrm{Pa}^{-1}[15]$ and is shown as a black line. In Figure $1 \mathrm{~d}$ it can be seen that after 1 pass of paint, the coating is opacity limited, where the surface emissivity is that of the combined surface and paint coating, resulting in a reduced thermoelastic response. The coating acts as a high emissivity coating at a thickness of 2 to 3 passes, at low frequencies ( 5 to $20 \mathrm{~Hz}$ ).

The results show that changes in the thermoelastic constant due to plastic deformation in stainless steel are measurable, despite the small magnitude of the thermoelastic response. The effects of paint coating provide a major difficulty in this work; if it was possible to ensure a uniform and accurate coating, variations caused by signal attenuation could be reduced, yielding a clearer understanding of the effects of plastic deformation. Further work will focus on fully investigating the effects of the 
paint coating, specifically for stainless steel, and on applying large amounts of compressive and tensile plastic deformation, defining the thermoelastic constant, $K$, for a large plastic strain range.

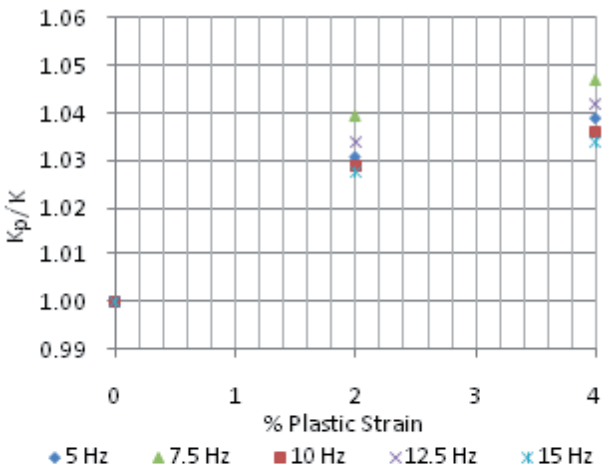

(a)

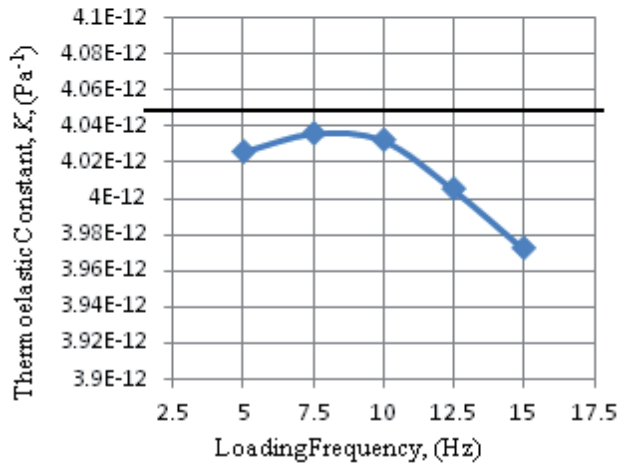

(c)

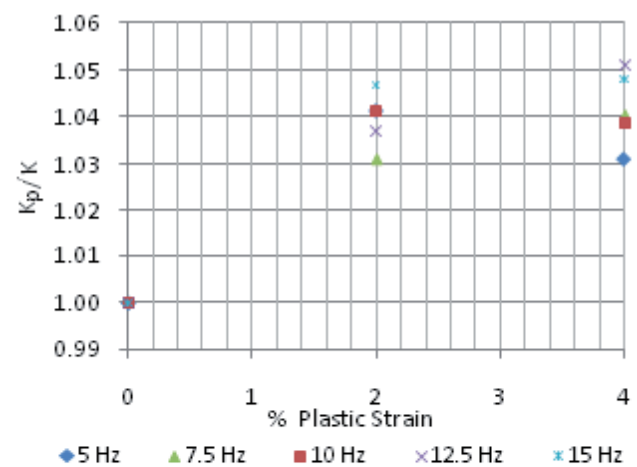

(b)

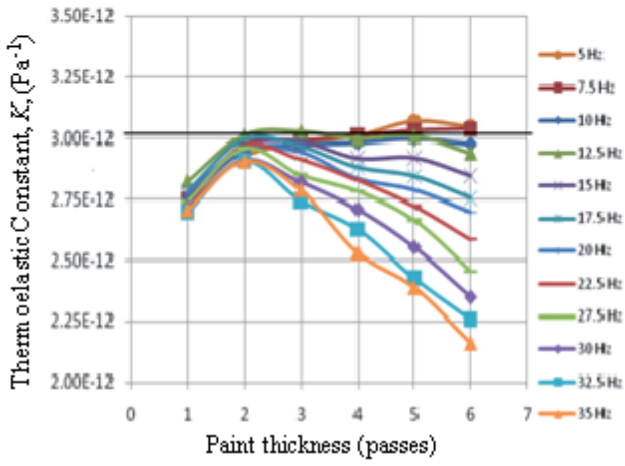

(d)

Figure 1: (a) Normalized thermoelastic constant with plastic strain at different loading frequencies in 316L (2 passes); (b) (3 passes); (c) Change in thermoelastic constant with loading frequency in 316L; (d) Change in thermoelastic constant with loading frequency and paint thickness in AISI 1016 steel.

\section{Assessment of cold expanded holes in aluminium plate}

The objective of this section is to investigate the small variations in thermoelastic response due to the presence of residual stress in a component containing representative residual stresses. The cold expansion technique is commonly used in aerospace applications as described in [17], as a means of enhancing fatigue life by delaying the initiation and propagation of fatigue cracks around holes. A large compressive residual stress is formed close to the hole, and reduces with distance from the hole; a tensile residual stress can be found much further from the hole due to the self-equilibrating stress field. There is a relatively well defined residual stress distribution around cold expanded holes; this provides an opportunity to examine departures in thermoelastic data, and assess the feasibility of applying a TSA based approach for residual stress analysis to actual components.

Four aluminium 7085-T7651 plates of dimensions $300 \mathrm{~mm} \times 150 \mathrm{~mm} \times 10 \mathrm{~mm}$ with $5 / 8$ inch holes were used. The holes were cold expanded to different levels $(0 \%, 2 \%$ and $4 \%)$ and reamed to a nominal diameter of $15.875 \mathrm{~mm}$; one hole was cold expanded to $4 \%$ and left unreamed. Tensile tests revealed that AL7085 exhibits only 1.5\% strain hardening, and it is recognised that this may not cause sufficient modification to the material properties. Each plate was coated with 2 passes of RS matt black paint to ensure a uniform high emissivity surface for TSA. An Instron 8800 servo- 
hydraulic test machine was used to cyclically load the plates (in the $300 \mathrm{~mm}$ direction) at a load amplitude of $\pm 10 \mathrm{kN}$ about a mean load of $15 \mathrm{kN}$; thermoelastic data was recorded at loading frequencies of 5 to $15 \mathrm{~Hz}$ in increments of $2.5 \mathrm{~Hz}$. Data was recorded from both the cold expansion mandrel entry and exit sides of the plates; The mandrel split was aligned with the top of the hole. A stand-off distance of $150 \mathrm{~mm}$ was used, focusing on the area adjacent to the hole.

The thermoelastic response around the cold expanded holes (4\%, 2\% and 0\%) for AL7085 is shown in Figure 2; one line shows the thermoelastic response, $\Delta T$, on a horizontal line taken across the hole (the data from the hole itself has been removed and can bee seen between approximately 12.5 and $28 \mathrm{~mm}$ ); a second line shows the background thermoelastic response away from the hole. The background response is similar $\left(0.045^{\circ} \mathrm{C}\right)$ across each specimen, however there is still a substantial level of noise. As the changes in thermoelastic response are significantly lower than can be expected in a typical TSA campaign, the level of noise shown here must be considered significant.

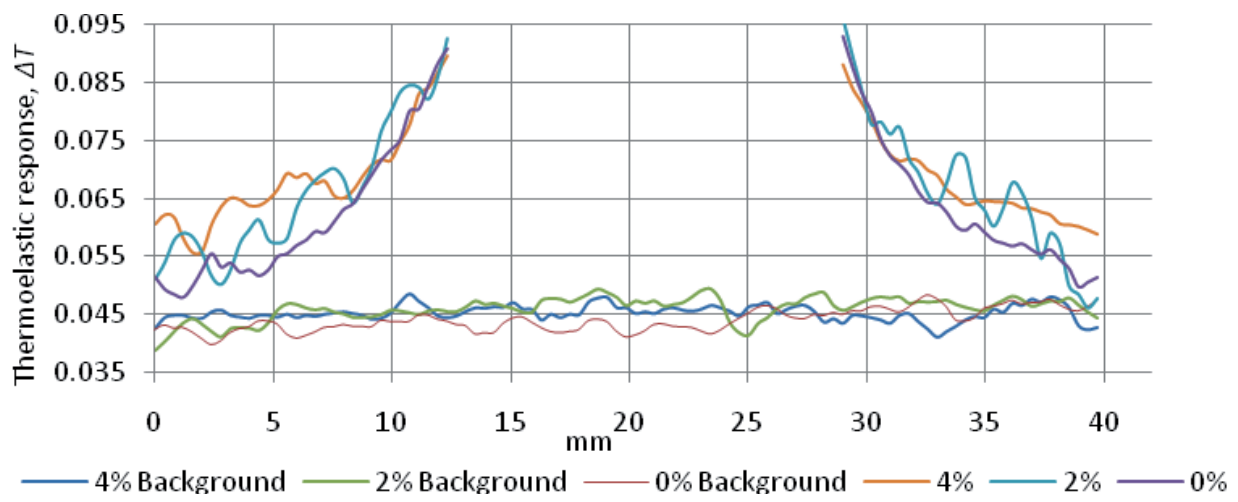

Figure 2: Thermoelastic response across cold expanded holes for AL7085-T7651 (10 Hz)

Away from the hole there is a clear difference in the thermoelastic response between the different levels of cold expansion, which is larger than the difference in background response of the three plates. This would be an expected characteristic since the larger the amount of cold expansion, the greater the residual stress around the hole. Interestingly for the $4 \%$ strain the difference is marked and indicates that the residual stress is penetrating more deeply into the material surrounding the hole. However, it should be noted that this may be as a result of the mean stress change and not a modification to the thermoelastic constant.

\section{Conclusions and future work}

The work presented in this paper details the promising initial steps that have been taken to define a methodology for using a TSA based approach for measuring residual stresses, based on the change in thermoelastic constant due to plastic deformation. It has been shown that plastic strain causes a change in thermoelastic constant for stainless steel. For metallic materials, the sensitivity to paint coating is shown to be large, and if specimens are not prepared with great care, the variations can be larger than the changes in thermoelastic constant that are of interest. It is vitally important that the surface coating applied to metallic specimens is uniform and has a high emissivity; the thickness of the coating is also a source of significant attenuation. Significant work has been undertaken to 
establish a methodology for the preparation of steel specimens for TSA, subsequently, suitable paint thickness and loading conditions have been defined.

A study on aluminum alloy plates with cold expanded holes demonstrated that there was a discernable difference in the response for a plate that has a $4 \%$ plastic strain. This is despite the material not being able to strain harden and potential variation in the paint coating from plate to plate. Future work will establish if the change in the response is due to a change in thermoelastic constant or the mean stress effect. Cold expanded holes in AL2024-T351 plates will be analysed in future work as this material strain hardens by approximately $29 \%$.

From the work described in the paper a number areas have been identified that warrant further investigation: (i) the effect of variations in the paint coating across the specimen, (ii) the effect of different substrate material and its interaction with the paint coating, (iii) a means of providing a uniform coating from specimens to specimen to provide a comparable 'background' response, (iv) accurately identifying identical positions from data set to data set.

\section{Acknowledgements}

The authors would like to acknowledge Airbus for the provision of the aluminum alloy and manufacture of the plates with cold expanded holes.

\section{References}

1. Dulieu-Barton, J.M. and Stanley, P., J. Strain Analysis, 33, 93-104 (1998)

2. Wong, A.K., Sparrow, J.G. and Dunn, S., J. Phys and Chem of Solids, 49, 395-400 (1988)

3. Robinson, A.F., Dulieu-Barton, J.M., Quinn, S. and Burguete, R.L., Proceedings of 7th International Conference on Modern Practices in Stress and Vibration Analysis, Journal of Physics: Conference Series 181 012029, doi:10.1088/1742-6596/181/1/012029. (2009)

4. Wong, A.K., Dunn, S.A., and Sparrow, J.G., Nature, 332, 613-615 (1988)

5. Patterson, E., Du, Y., and Backman, D., Proc. SEM XI, Orlando, FL. (2008)

6. Quinn, S., Dulieu-Barton, J.M., and Langlands, J.M., Strain, 40, 127-133 (2004)

7. McKelvie, J., Proceedings of SPIE, 731, 44-53 (1987)

8. Rosenholtz, J., and Smith, D., J. App. Physics, 21, 396-399 (1950)

9. Rosenfield, A.R. and Averbach, B.L., J. App. Physics, 27, 154-156 (1956)

10. Eaton-Evans, J.M., Dulieu-Barton, J.M., Little, E.G. and Brown I.A., Journal of Strain Analysis for Engineering Design, 41, 481-495 (2006)

11. Dulieu-Barton, J.M. and Quinn, S., Journal of Strain Analysis, 37, 59-71 (2002)

12. Matweb, Online Materials Resource for Material Property Data, www.matweb.com, [20/01/10]

13. Quinn, S., Dulieu-Barton, J.M., Eaton-Evans, J.M., Fruehmann, R.K., and Tatum, P.J, J. Strain Analysis, 43, 451-468 (2008)

14. Eaton-Evans, J., PhD thesis University of Limerick (2006)

15. Robinson, A.F., Dulieu-Barton, J.M., Quinn, S. and Burguete, R.L., Submitted: Measurement Science and Technology. (2010)

16. Welch, C.S. and Zickel, M.J., Review of Progress in Quantitative Nondestructive Evaluation, 12, 1923-1929 (1993)

17. Ozdemir, A.T., and Edwards, L., J. Strain Analysis, 31, 413-421 (1996) 